\title{
EVALUACIÓN DE LA USABILIDAD PERCIBIDA DE GOOGLE CLASSROOM, DRIVE Y MEET EN EL PROCESO DE ENSEÑANZA Y APRENDIZAJE DE LA UNIVERSIDAD NACIONAL DE SAN ANTONIO Abad del CUSCO DURANTE LA PANDEMIA DEL COVID-19
}

\author{
JISBAJ GAMARRA SALAS \\ jisbaj.gamarra@unsaac.edu.pe / ORCID: 0000-0001-8526-5005 \\ William Alberto Chávez Espinoza \\ william.chavez@unsaac.edu.pe / ORCID: 0000-0002-7170-8837 \\ LISETH URPY SEgUNDO CARPIO \\ liseth.segundo@unsaac.edu.pe / ORCID: 0000-0001-7985-7477
}

Departamento Académico de Ingeniería Informática Universidad Nacional de San Antonio Abad del Cusco, Perú

El Perú, como el resto de países del mundo, ha sido afectado en muchos sentidos por la pandemia generada por el COVID-19. El sector educación no fue ajeno a esta situación. Las clases presenciales en todos los niveles de educación fueron canceladas y se migró a las clases virtuales. En este contexto, las aplicaciones educativas virtuales tomaron mayor protagonismo en los procesos de enseñanza y aprendizaje, siendo la usabilidad un atributo importante que debe ser considerado en este tipo de plataformas. El objetivo de esta investigación es medir la usabilidad de las principales aplicaciones educativas virtuales que actualmente utiliza la Universidad Nacional de San Antonio Abad del Cusco en su proceso de enseñanza y aprendizaje: Google Classroom, Google Drive y Google Meet. Para medir la usabilidad de estas aplicaciones se aplicó el cuestionario System Usability Score (SUS) a una población aproximada de 1150 docentes y 19000 estudiantes de los cuales respondieron un total de 211 participantes, 137 alumnos y 74 docentes. El estudio es de enfoque cuantitativo y de alcance descriptivo con muestreo no probabilístico. El resultado del estudio muestra que la usabilidad percibida para las tres aplicaciones en general es buena para todos los participantes (promedio de puntuación SUS 69,85). En ese sentido, considerando el contexto 
de la pandemia mundial, el uso de las herramientas de Google es conveniente para adaptarse rápidamente al proceso de enseñanza en el ámbito virtual y dichas herramientas son fáciles de usar y aprender.

PALABRAS CLAVE: usabilidad / Google Classroom / Google Driv / Google Meet / escala SU / capacidad de aprendizaje

\section{PERCEIVED USABILITY EVALUATION OF GOOGLE CLASSROOM, DRIVE AND MEET IN THE TEACHING AND LEARNING PROCESS AT THE UNIVERSIDAD NACIONAL DE SAN ANTONIO ABAD DEL CUSCO DURING THE COVID-19 PANDEMIC}

\section{Abstract}

Perú, like the rest of the world, has been affected in many ways by the COVID -19 . The education sector was not immune to this situation. Face-to-face classes at all levels of education were canceled, education had to migrate to virtual classes. In this context, virtual educational applications took a more significant role in the teaching and learning processes, usability a critical attribute that must be considered in this platform. This research aims to measure the usability of the leading educational virtual applications currently used by the National University of San Antonio Abad del Cusco in its teaching and learning process: Google Classroom, Google Drive, and Google Meet. The study has a quantitative approach and descriptive scope with non-probabilistic sampling. To measure the usability of these applications, the System Usability Score (SUS) questionnaire was applied to an approximate population of 1150 teachers and 19000 students, of which a total of 211 participants, 137 students, and 74 teachers responded. The study results show that the perceived usability for the three applications, in general, is good for all the participants (SUS score 69,85). The usability analyzed in detail is very good for teachers, and learning is ideal for both. In this sense, considering the context of the global pandemic, Google tools are convenient to quickly adapt to the teaching process in the virtual environment, and these tools are easy to use and learn.

KEYWORDS: usability / Google Classroom / Google Driv / Google Meet / SUS scale / learnability 


\section{INTRODUCCIÓN}

Debido al COVID-19, todos los países del mundo han decidido cerrar las instituciones educativas para prevenir los contagios (Ahmad et al., 2020; Dash et al., 2021; Organización de las Naciones Unidas para la Educación [UNESCO], 2021). En ese sentido, en el Perú, a partir del 11 de marzo del 2020, como medidas de prevención y control del COVID-19, se dictó una serie de decretos supremos que declararon en estado de emergencia sanitaria a nivel nacional. Estas medidas, respecto al tema educativo, señalan que las instituciones públicas y privadas que brinden el servicio educativo en cualquiera de sus niveles deben suspender sus actividades presenciales (Plataforma digital única del Estado Peruano, 2021).

Las instituciones educativas estuvieron forzadas a migrar a un entorno virtual de aprendizaje en línea y a distancia. Las aplicaciones educativas como Zoom, Google Classroom, Skype, entre otros, son las más usadas para migrar todo al entorno virtual (Ahmad et al., 2020). Sin embargo, la migración no es fácil y requiere de competencias en tecnologías informáticas y adaptación de prácticas pedagógicas a un contexto virtual, tanto para docentes como para alumnos. En ese sentido, muchas instituciones educativas están en proceso de adaptación e integración de los procesos de enseñanza-aprendizaje presencial al entorno virtual y sostener este proceso de la mejor manera (Akcil et al., 2021). Además, el proceso no es fácil puesto que en algunas zonas que no tienen acceso a internet la adaptación es compleja o nula. También, tanto profesores como estudiantes no estaban preparados para afrontar el uso de herramientas digitales para el entorno de enseñanza-aprendizaje que les era requerido en el contexto de esta emergencia sanitaria (Bardales et al., 2020).

Después de un crecimiento sin precedentes en la educación superior en las últimas décadas, la educación de más de 220 millones de estudiantes de nivel superior se interrumpió repentinamente en el año 2020 por el cierre de los locales debido al COVID-19 (UNESCO, 2021).

La Universidad Nacional de San Antonio Abad del Cusco (Unsaac), como muchas de las universidades del Perú, tuvo que migrar el proceso de enseñanza y aprendizaje al entorno virtual para no detener las actividades de aproximadamente 3000 asignaturas programadas por semestre $y$, consecuentemente, perjudicar a 19000 estudiantes, 1150 profesores (Nina et al., 2017; Supertintendencia Nacional de Educación Superior Universitaria [Sunedu], 2019). La Unsaac optó por utilizar herramientas de Google para la educación (Google Classroom, Drive y Meet) que estaban disponibles desde el año 2013, pero que no eran utilizadas completamente por toda la universidad. En 2018, solo el $18 \%$ de 30000 cuentas de usuarios usaban de manera frecuente dichas herramientas (Nina, 2019). Es decir, más del $80 \%$ de los miembros de la comunidad universitaria no estaban preparados para hacer uso de las herramientas de Google en el proceso de aprendizaje. 
En este contexto, conocer el grado de complejidad de este proceso de adaptación, desde el punto de vista de la usabilidad de estas herramientas, se convierte en un factor clave.

La usabilidad es un atributo de calidad que mide lo fácil que puede llegar a ser el uso de las interfaces gráficas de usuario de un sistema y no puede verse como una propiedad de una sola dimensión pues incluye cinco atributos: capacidad de aprendizaje, eficiencia, memorabilidad, errores y satisfacción (Nielsen, 1994).

Para medir la usabilidad existen varios métodos de evaluación como encuestas o cuestionarios, pruebas de usuario, evaluación heurística, entrevistas, entre otros. Según Paz y Pow-Sang (2016), en su estudio de mapeo sistemático de métodos de evaluación de usabilidad, se reporta con mayor frecuencia la técnica de los cuestionarios. En ese sentido, existen varias técnicas de cuestionarios y entre ellas tenemos a SUS (System Usability Scale) que se ha convertido en un estándar de la industria, con referencias en más de 1300 artículos y publicaciones (Usability.gov, 2021).

Esta investigación tiene por objetivo evaluar la usabilidad percibida, desde la perspectiva de estudiantes y profesores, de Google Classroom, Drive y Meet en el proceso de enseñanza y aprendizaje de la Unsaac durante la pandemia del COVID-19 utilizando SUS.

Utilizaremos SUS por ser una técnica de rápida aplicación y de amplia utilización (Brooke, 1995).

El documento está distribuido de la siguiente manera: en la segunda sección se describen los importantes antecedentes del estudio, en la tercera sección se presenta la metodología, en la cuarta sección los resultados y, finalmente, en la quinta sección se presentan las discusiones y conclusiones del estudio.

\section{ANTECEDENTES}

\subsection{Herramientas de Google en procesos de aprendizaje}

Según Nina et al. (2017) la suite de Google for Education es un conjunto de aplicaciones en línea útiles para la comunicación y colaboración entre profesores y estudiantes en organizaciones educativas. Las herramientas disponibles en la suite de Google son: Gmail, Google Hangout, Google Meet, Google Drive, Google Form, Google Site, Google Classroom, Calendar, Grupos de Google, entre otros. Además, la suite de Google está integrada a las aplicaciones externas como Youtube, Google Chrome, Blogger, y Búsquedas en Google (Google for Education, 2021).

Las herramientas de la suite de Google más utilizadas en la educación son Google Drive, Classroom y Meet (Adkins et al., 2012; Akcil et al., 2021; Rusmansyah et al., 2021; Nina et al., 2017). 
Google Drive permite almacenar cualquier tipo de archivo en la nube de Google y crear documentos de Google, hojas de cálculo, formularios, sitios, entre otros. Así mismo, Google Drive dispone de funcionalidades para que un grupo de usuarios pueda compartir y colaborar en línea. Por ejemplo, con Google Sites se pueden crear sitios web de grupos seguros en los que pueden compartir y trabajar en documentos de manera colaborativa (Lindoo, 2009). Así mismo, con documentos de Google, un grupo de usuarios puede crear una presentación de diapositivas para lograr un aprendizaje activo en el que todos pueden colaborar e interactuar (Adkins, 2012).

En segundo lugar, Google Classroom, desde su aparición en el año 2014, ha ido creciendo constantemente hasta consolidarse como la herramienta por excelencia de Google para facilitar el proceso de enseñanza, aprendizaje y la comunicación entre profesores y estudiantes. Así mismo, entre sus principales funcionalidades está crear aulas digitales, publicar material digital didáctico, publicar anuncios, asignar y entregar tareas, realizar comentarios, gestionar horarios y realizar la calificación de tareas con la posibilidad de utilizar rúbricas. Además, Google Classroom es una plataforma de aprendizaje en línea gratuita con varias funciones que respaldan el aprendizaje interactivo, divertido y significativo con una gestión del aula flexible. Por ejemplo, Google Classroom puede ayudar a los profesores de ciencias a administrar clases, realizar actividades de laboratorio y realizar evaluaciones en línea (Widiyatmoko, 2021). Finalmente, Google Classroom se combina con Google Drive para la distribución de tareas, videos y uso compartido de archivos de presentaciones, y Google Calendar para programar el calendario de reuniones.

En tercer lugar, Google Meet es una herramienta de videoconferencias con funcionalidades para crear e invitar a reuniones en línea, compartir pantalla, mensajería, diseños para visualización, grabar reuniones, traducciones automáticas de idiomas, entre otros. En ese sentido, Rusmansyah et al. (2021) señala que Google Meet facilita la interacción entre profesores y estudiantes como si estuvieran aprendiendo en clase directamente y para Septantiningtyas et al. (2021) Google Meet influye de manera significativa en la motivación de aprendizaje de los estudiantes, puesto que los estudiantes consideran que es fácil de implementar y permite la flexibilidad en tiempo y espacio.

Finalmente, existen otras herramientas de Google que destacan en el proceso de enseñanza y aprendizaje, como por ejemplo Gmail, Grupos de Google y Google Hangouts que disponen de funcionalidades para organizar mensajes, correo, listas de distribución, organizar eventos y mensajería instantánea. Por ejemplo, el uso de Google Hangouts ha permitido una comunicación rápida y fácil en temas de consejería, asesoramiento y tutoría en organizaciones educativas (Mendoza, 2015). Por su parte, Google Calendar permite gestionar y compartir eventos de manera fácil y con la posibilidad de sincronizarlos con dispositivos móviles (Lewis y Kimmel-Smith, 2011). 
En la tabla 1, se muestra que las herramientas de Google pueden aplicarse a modelos de actividades docentes de Gagne y ayudan a garantizar la integración de la tecnología en el aula (Akcil et al., 2021).

Tabla 1

Modelo de actividades docentes de Gagne y Google Workspace Tools

\begin{tabular}{|c|c|c|c|}
\hline Etapa & $\begin{array}{l}\text { Nueve etapas de la enseñanza } \\
\text { del modelo de Gagne }\end{array}$ & Logo & $\begin{array}{l}\text { Actividades a realizar y } \\
\text { herramientas web adecuadas } \\
\text { (ejemplo) }\end{array}$ \\
\hline \multirow{4}{*}{ INTRODUCCIÓN } & & & $\begin{array}{l}\text { Presentación visual - Fotos de } \\
\text { Google }\end{array}$ \\
\hline & $\begin{array}{l}\text { 1. Captar la atención } \\
\text { 2. Informar sobre el objetivo }\end{array}$ & & Mostrar videos - YouTube \\
\hline & $\begin{array}{l}\text { 3. Asociación con } \\
\text { aprendizaje previo }\end{array}$ & $\begin{array}{l}\text { Google } \\
\text { AR\&VR }\end{array}$ & $\begin{array}{l}\text { Uso de la simulación - Google AR } \\
\text { y VR }\end{array}$ \\
\hline & & & $\begin{array}{l}\text { Mostrar tarjeta de información - } \\
\text { Google Keep }\end{array}$ \\
\hline \multirow{11}{*}{ DESARROLLO } & \multirow{11}{*}{$\begin{array}{l}\text { 4. Presentación del contenido } \\
\text { 5. Proporcionar orientación } \\
\text { 6. Rendimiento revelador } \\
\text { 7. Proporcionar comentarios }\end{array}$} & & Investigar: Google Scholar - Chrome \\
\hline & & & $\begin{array}{l}\text { Preparar una presentación - Google } \\
\text { Slides }\end{array}$ \\
\hline & & & $\begin{array}{l}\text { Interacción en el aula virtual - } \\
\text { Google Classroom }\end{array}$ \\
\hline & & & $\begin{array}{l}\text { Reunir los contenidos del curso - } \\
\text { Google Sites }\end{array}$ \\
\hline & & & Hacer uso de infografías - Google \\
\hline & & & Drawing \\
\hline & & & Conferencia en vivo - Google Meet \\
\hline & & & Mensajería - Gmail \\
\hline & & & $\begin{array}{l}\text { Uso de mapas mentales - Google } \\
\text { Mindmap (Chrome Add-on) }\end{array}$ \\
\hline & & Q & Tener discusiones - Google Groups \\
\hline & & & $\begin{array}{l}\text { Documentos de prácticas } \\
\text { interactivas - Google Jamboard }\end{array}$ \\
\hline
\end{tabular}


(continuación)

\begin{tabular}{|c|c|c|c|}
\hline \multirow{5}{*}{ EVALUACIÓN } & \multirow{5}{*}{$\begin{array}{l}\text { 8. Evaluación del desempeño } \\
\text { 9. Asegurar el desempeño } \\
\text { de lo aprendido y } \\
\text { fortalecer su transferencia }\end{array}$} & $\Delta$ & $\begin{array}{l}\text { Almacenamiento de contenido } \\
\text { educativo - Google Drive }\end{array}$ \\
\hline & & Google & $\begin{array}{l}\text { Estudios comunes - Google } \\
\text { Documents }\end{array}$ \\
\hline & & := & Cuestionario - Google Forms \\
\hline & & $\begin{array}{l}\text { Edu } \\
\text { lastic }\end{array}$ & $\begin{array}{l}\text { Crear prueba en línea - Google } \\
\text { Edulastic }\end{array}$ \\
\hline & & 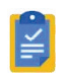 & $\begin{array}{l}\text { Dar tarea y retroalimentación - } \\
\text { Google HomeWorks }\end{array}$ \\
\hline
\end{tabular}

Fuente: Adaptado de Akcil et al. (2021)

\subsection{La usabilidad y sus métodos de evaluación}

De acuerdo con Jakob Nielsen (1994), la usabilidad es un atributo de calidad del software que mide lo fácil que puede llegar a ser el uso de las interfaces de usuario de un sistema, analizados a partir de los siguientes atributos:

- Aprendizaje: fácil de aprender para los inexpertos.

- Eficiencia: eficiente para usuarios expertos.

- Facilidad de memorizar: fácil de recordar lo aprendido por usuarios ocasionales.

- Errores: frecuencia de errores y qué tan graves son.

- Satisfacción: que tan agradable es el diseño para usar.

De acuerdo con Paz y Pow-Sang (2016), para evaluar la usabilidad de una aplicación de software se reportan con mayor frecuencia las técnicas de cuestionarios, pruebas de usuario, evaluación heurística, entrevista y pensar en voz alta. Véase la tabla 2.

\section{Tabla 2}

Frecuencia de uso de cada método de evaluación de usabilidad

\begin{tabular}{lcc}
\hline Método de evaluación de usabilidad & $\begin{array}{c}\text { Número de veces que fue } \\
\text { utilizado el método }\end{array}$ & Porcentaje \\
\hline Survey / Questionnaire & 104 & 26,26 \\
User Testing & 56 & 14,14 \\
Heuristic Evaluation & 50 & 12,63 \\
Interview & 41 & 10,35 \\
\hline
\end{tabular}




\begin{tabular}{lcc} 
(continuación) & 38 & \\
\hline $\begin{array}{l}\text { User Testing - Thinking Aloud / } \\
\text { Thinking Out Loud }\end{array}$ & 19 & 4,80 \\
Software Metrics / Usability Metrics & 16 & 4,04 \\
Automated Evaluation via Software & 11 & 2,78 \\
Tool & 11 & 2,78 \\
Cognitive Walkthrough & 6 & 1,52 \\
Prototype Evaluation & 5 & 1,26 \\
Focus Group & 5 & 1,26 \\
Checklist Verification & 34 & 8,59 \\
Pencil y Paper & & \\
Others & 5 &
\end{tabular}

Fuente: Paz y Pow-Sang (2016)

Según Cáceres y Pow-Sang (2018), la técnica de cuestionario para evaluar la usabilidad es un conjunto de preguntas específicas que responde una cantidad representativa de usuarios luego de utilizar una aplicación de software. Entre los tipos de cuestionarios más utilizados se encuentran:

- SUMI (Software Usability Measurement Inventory)

- SUS (System Usability Scale)

- CSUQ (Computer System Usability Questionnaire)

- PUTQ (Purdue Usability Testing Questionnaire)

- PSSUQ (Post-Study System Usability)

- UEQ (User Experience Questionnaire)

- UES (User Engagement Scale)

- QUIS (Questionnaire for User Interface Satisfaction)

Considerando el estudio de Cáceres y Pow-Sang (2018), en la presente investigación se hace uso del cuestionario SUS (System Usability Scale) por ser uno de los más utilizados entre las técnicas de tipo cuestionario.

\subsection{System Usability Scale (SUS)}

Existen diferentes estudios de evaluación de la usabilidad de sistemas de gestión del aprendizaje a través de técnicas como cuestionarios, pruebas de usabilidad, escalas, encuestas e incluso técnicas de minería de datos (Al-Omar, 2018). 
Incidiremos en la Escala de Usabilidad del Sistema (SUS, del inglés System Usability Scale ) que es la técnica utilizada para el caso de estudio.

SUS es un cuestionario simple de diez preguntas desarrollado por John Brooke en 1986 como respuesta a la demanda por encontrar técnicas que se administren de forma rápida y sencilla, pero lo suficientemente fiables, en comparación con los métodos que demandan largos periodos de tiempo que generan frustración en los usuarios (Brooke, 1995). Permite evaluar la usabilidad en diferentes productos y servicios, incluyendo sitios web, hardware, software, dispositivos móviles y aplicaciones y se ha convertido en un estándar con más de 1300 referencias en publicaciones y artículos (Usability.gov, 2021).

SUS usa la escala de Likert con cinco respuestas para cada pregunta que van desde "totalmente en desacuerdo" hasta "totalmente de acuerdo" (Brooke, 1995).

\subsection{Estudios de usabilidad de Google Meet, Drive y Classroom}

La usabilidad se convierte en un factor crítico para las aplicaciones educativas (Cáceres et al., 2018). En ese sentido, a continuación presentaremos algunos trabajos de investigación que se han realizado en dicha área.

En Mavri y Hadjiconstantinou (2018) se evalúa la usabilidad de dos tecnologías colaborativas de aprendizaje, el Moodle Elgg y el Google Drive, así como su impacto pedagógico en términos de resultados de aprendizaje percibidos por los estudiantes. El estudio reclutó a un total de 28 estudiantes quienes fueron consultados y evaluados sobre el nivel de facilidad con que los usuarios realizan las tareas, la ocurrencia de errores y la experiencia de cada usuario. Los resultados mostraron que, en términos de usabilidad, el Google Drive es significativamente mejor que Moodle Elgg en cuanto a su facilidad de uso y experiencia de usuario.

La plataforma educativa Google Classroom también ha sido evaluada en términos de usabilidad y satisfacción del usuario según De Campos Filho (2019). Para este estudio se utilizó el cuestionario de Attrakdiff en un total de 110 estudiantes universitarios. El análisis de satisfacción de los usuarios mostró que esta plataforma resulta adecuada para el proceso de aprendizaje.

Finalmente, de acuerdo con Dash et al. 2021), se estudia la usabilidad y practicidad de las plataformas de enseñanza en línea y su importancia en el periodo de la pandemia del COVID-19. Fueron comparadas un total de siete plataformas (Zoom, Google Meet, Google Classroom, Microsoft Teams, Cisco Webex, Go ToMeet y Say Namaste) resaltando sus características esenciales, beneficios, interfaz de usuario y limitaciones. Los resultados mostraron que las plataformas mencionadas anteriormente facilitan la realización de conferencias educativas (reuniones virtuales) y que son ideales en términos de usabilidad, salvando lógicamente sus diferencias. 


\section{METODOLOGÍA}

La investigación tiene un enfoque cuantitativo y alcance descriptivo con muestreo no probabilístico. La metodología de este estudio se lleva a cabo en dos pasos: primero, se aplica el cuestionario SUS a docentes y alumnos de la Unsaac al finalizar el semestre académico 2021-I entre el 18 de marzo y el 7 de mayo del 2021, para tres de los softwares más utilizados de la Google Suite for Education: Google Drive, Google Classroom y Google Meet; segundo, se calcula el puntaje SUS para cada aplicación y rol de usuario (docente y alumno).

En base a la los datos de Sunedu (2019), la población de estudio es aproximadamente de 1150 docentes y 19000 estudiantes. En ese sentido, la investigación realiza un muestreo no probabilístico por conveniencia de 211 participantes, de los cuales, 137 son alumnos y 74 son docentes. Según Hernández et al. (2010) el muestreo no probabilístico, también llamado muestras dirigidas, supone un procedimiento de selección informal y se utiliza en diversas investigaciones cuantitativas y cualitativas. La aplicación de la encuesta se realizó online utilizando Google Form para evitar riesgos a los investigadores y entrevistados por la pandemia del COVID-19.

\subsection{Cuestionario SUS}

El cuestionario SUS está formado por diez preguntas básicas (Brooke, 1995):

Tabla 3

Cuestion preguntas de SUS

\begin{tabular}{ll}
\hline ID & Enunciado de la pregunta \\
\hline P1 & Creo que usaría este sistema frecuentemente. \\
P2 & Encuentro este sistema innecesariamente complejo. \\
P3 & Creo que el sistema fue fácil de usar. \\
P4 & Creo que necesitaría ayuda de una persona con conocimientos técnicos para usar este \\
& sistema. \\
P5 & Las funciones de este sistema están bien integradas. \\
P6 & Creo que el sistema es muy inconsistente. \\
P7 & Imagino que la mayoría de la gente aprendería a usar este sistema en forma muy rápida. \\
P8 & Encuentro que el sistema es muy difícil de usar. \\
P9 & Me siento confiado al usar este sistema. \\
P10 & Necesité aprender muchas cosas antes de ser capaz de usar este sistema. \\
\hline
\end{tabular}

Fuente: adaptado de (Brooke, 1995) 
Sobre la base de estas preguntas se crea un cuestionario para cada aplicación a evaluar, reemplazando la palabra "sistema" por el nombre correspondiente (Google Classroom, Google Drive o Google Meet).

Cada pregunta tiene una escala de uno a cinco puntos, siendo uno equivalente a totalmente en desacuerdo y cinco a totalmente de acuerdo. Hay cinco declaraciones positivas y cinco negativas, que se alternan, las impares son positivas y las pares negativas.

\subsection{Cálculo del SUS score}

Para el cálculo de los resultados hay que diferenciar entre la escala de puntuación y la escala de contribución de cada sentencia. La puntuación, como se mencionó anteriormente, es una escala de Likert que va de uno a cinco, mientras que la contribución va de cero a cuatro.

La contribución se calcula en base al tipo de pregunta. La contribución para las preguntas positivas se obtiene restando 1 a la puntuación del usuario (ver Ecuación 1), mientras que para las negativas se obtiene restando el valor de la puntuación del usuario a 5 (ver Ecuación 2). Luego, multiplicar la suma de las contribuciones por 2,5 para obtener el puntaje SUS en el rango de 0 a 100 (Brooke, 1995).

$$
\text { SUS } \text { impar }=\left(\sum_{i=1}^{10} \text { puntuación }-1\right) * 2.5 ; \text { dónde i es impar }
$$

Ecuación 1: cálculo SUS para pregunta impar

$$
S U S_{\text {par }}=\left(\sum_{i=1}^{10} 5-\text { puntuación }\right) * 2.5 ; \text { dónde i es par }
$$

Ecuación 2: cálculo SUS para pregunta par

El SUS por sí mismo resulta difícil de interpretar; es decir, la puntuación obtenida en el rango de 0 a 100 no se puede definir si es buena o mala porque no se cuenta con ningún tipo de comparación. Sin embargo, con el tiempo, por la cantidad de estudios realizados, se han obtenido grandes volúmenes de datos que motivaron a varios autores a desarrollar escalas de calificación para su interpretación (Lewis y Sauro, 2018), como por ejemplo el grado de calificación curva propuesta por Sauro et al. (2012).

Sauro (2018), describe cinco enfoques, de los más usados, que ayudan a interpretar el puntaje SUS de manera significativa. 
Tabla 4

Percentiles, grados, adjetivos y categorías de NPS para describir las puntuaciones de SUS

\begin{tabular}{|c|c|c|c|c|c|}
\hline Grados & $\begin{array}{l}\text { Puntaje } \\
\text { SUS }\end{array}$ & $\begin{array}{l}\text { Rango de } \\
\text { percentiles }\end{array}$ & Adjetivos & Aceptabilidad & NPS1 \\
\hline$A+$ & $84,1-100$ & $96-100$ & $\begin{array}{l}\text { Lo mejor } \\
\text { imaginable }\end{array}$ & Aceptable & Promotor \\
\hline A & $80,8-84,0$ & $90-95$ & Excelente & Aceptable & Promotor \\
\hline A- & $78,9-80,7$ & $85-89$ & Muy bueno & Aceptable & Promotor \\
\hline $\mathrm{B}+$ & $77,2-78,8$ & $80-84$ & Muy bueno & Aceptable & Pasivo \\
\hline B & $74,1-77,1$ & $70-79$ & Muy bueno & Aceptable & Pasivo \\
\hline$B-$ & $72,6-74,0$ & $65-69$ & Muy bueno & Aceptable & Pasivo \\
\hline $\mathrm{C}_{+}$ & $71,1-72,5$ & $60-64$ & Muy bueno & Aceptable & Pasivo \\
\hline C & $65,0-71,0$ & $41-59$ & Bueno & Marginal & Pasivo \\
\hline C- & $62,7-64,9$ & $35-40$ & Bueno & Marginal & Pasivo \\
\hline D & $51,7-62,6$ & $15-34$ & Bueno & Marginal & Detractor \\
\hline
\end{tabular}

Fuente: Sauro (2018)

Adicionalmente a la interpretación unidimensional del puntaje general del SUS, esta se puede descomponer en un análisis más detallado en base a dos subescalas, usabilidad y capacidad de aprendizaje (Lewis y Sauro, 2009).

Las sentencias cuatro y diez del cuestionario están alineadas con la escala de capacidad de aprendizaje y las otras ocho con la de usabilidad. Para el cálculo de sus contribuciones, la suma de sus puntuaciones se multiplica por 12,5 y 3,125 respectivamente, con el objetivo de utilizar las mismas escalas de comparación con que se interpreta la puntuación general del SUS (Lewis y Sauro, 2009).

\section{RESULTADOS}

\subsection{Descripción de la muestra}

Características de la participación de alumnos y docentes de un total de 211 participantes, de los cuales 137 son alumnos (64,92 \%) y 74 son docentes $(35,07 \%)$.

1 Net Prometers Score es una métrica que cuantifica cuántas personas probablemente recomienden un producto en comparación con aquellos que probablemente lo critiquen (Fessenden, 2016) 
Tabla 5

Características demográficas de alumnos

\begin{tabular}{|c|c|c|}
\hline \multirow{2}{*}{ Género (\%) } & Masculino & 62,04 \\
\hline & Femenino & 37,95 \\
\hline \multirow{3}{*}{ Edad (años) } & Máximo & 59 \\
\hline & Mínimo & 18 \\
\hline & Media & 25,066 \\
\hline \multirow{4}{*}{$\begin{array}{l}\text { Dispositivo } \\
\text { que utiliza (\%) }\end{array}$} & Móvil & 68,61 \\
\hline & Laptop & 54,01 \\
\hline & Pc de escritorio & 15,32 \\
\hline & Tablet & 3,64 \\
\hline \multirow{6}{*}{$\begin{array}{l}\text { Lugar de } \\
\text { acceso al } \\
\text { Internet (\%) }\end{array}$} & Desde la casa de un familiar o amigo & 18,24 \\
\hline & Desde su casa & 49,63 \\
\hline & $\begin{array}{l}\text { Utilizó el modem+ chip distribuido por la } \\
\text { Unsaac }\end{array}$ & 23,35 \\
\hline & Desde una cabina pública & 53,64 \\
\hline & Desde mi oficina / lugar de trabajo & 2,91 \\
\hline & Desde un lugar público con wifi & 2,18 \\
\hline
\end{tabular}

Elaboración propia

Tabla 6

Características demográficas de docentes

\begin{tabular}{|c|c|c|}
\hline \multirow{2}{*}{ Género (\%) } & Masculino & 68,91 \\
\hline & Femenino & 31,09 \\
\hline \multirow{3}{*}{ Edad (años) } & Máximo & 74 \\
\hline & Mínimo & 35 \\
\hline & Media & 54,31 \\
\hline \multirow{4}{*}{$\begin{array}{l}\text { Dispositivo } \\
\text { que utiliza } \\
(\%)\end{array}$} & Móvil & 21,62 \\
\hline & Laptop & 70 \\
\hline & Pc de escritorio & 14 \\
\hline & Tablet & 3 \\
\hline
\end{tabular}

(continúa) 
(continuación)

\begin{tabular}{llc}
\hline & Desde la casa de un familiar o amigo & 3 \\
& Desde su casa & 62 \\
Lugar de & Utilizó el modem+chip distribuido por la Unsaac & 23 \\
acceso al & Desde una cabina pública & 0 \\
Internet & Desde mi oficina/lugar de trabajo & 2 \\
& Desde un lugar público con Wifi & 1 \\
\hline
\end{tabular}

Elaboración propia

\subsection{Resultados de la usabilidad de Google Classroom, Drive y Meet}

La tabla 7 presenta los resultados de las respuestas a las preguntas de usabilidad obtenidas para las tres aplicaciones (Google Classroom, Google Drive y Google Meet). Se detalla el promedio y desviación estándar para cada pregunta, por aplicación y grupo de usuarios.

En la tabla 8 se muestran aplicadas las reglas de puntuación del SUS, los puntajes promedio de SUS y las escalas de calificación de adjetivos para Google Classroom, Google Drive Google y Google Meet, tanto para alumnos como para docentes. El promedio general para las tres aplicaciones para alumnos es de 69,70 (equivalente a una escala de adjetivo de bueno) y 70 (equivalente a una calificación de bueno) para docentes.

Los resultados del análisis de las subescalas del SUS se muestran en la tabla 9. En el caso de las tres aplicaciones, para los alumnos, en el factor de usabilidad se obtiene un promedio de 71,04 (escala de adjetivo bueno) y en el factor capacidad de aprendizaje de 64,14 (escala de adjetivo bueno) y para los docentes, en el factor de usabilidad se obtiene un promedio de 74,17 (escala de adjetivo muy bueno) y en el factor capacidad de aprendizaje de 53,27 (escala de adjetivo bueno). 


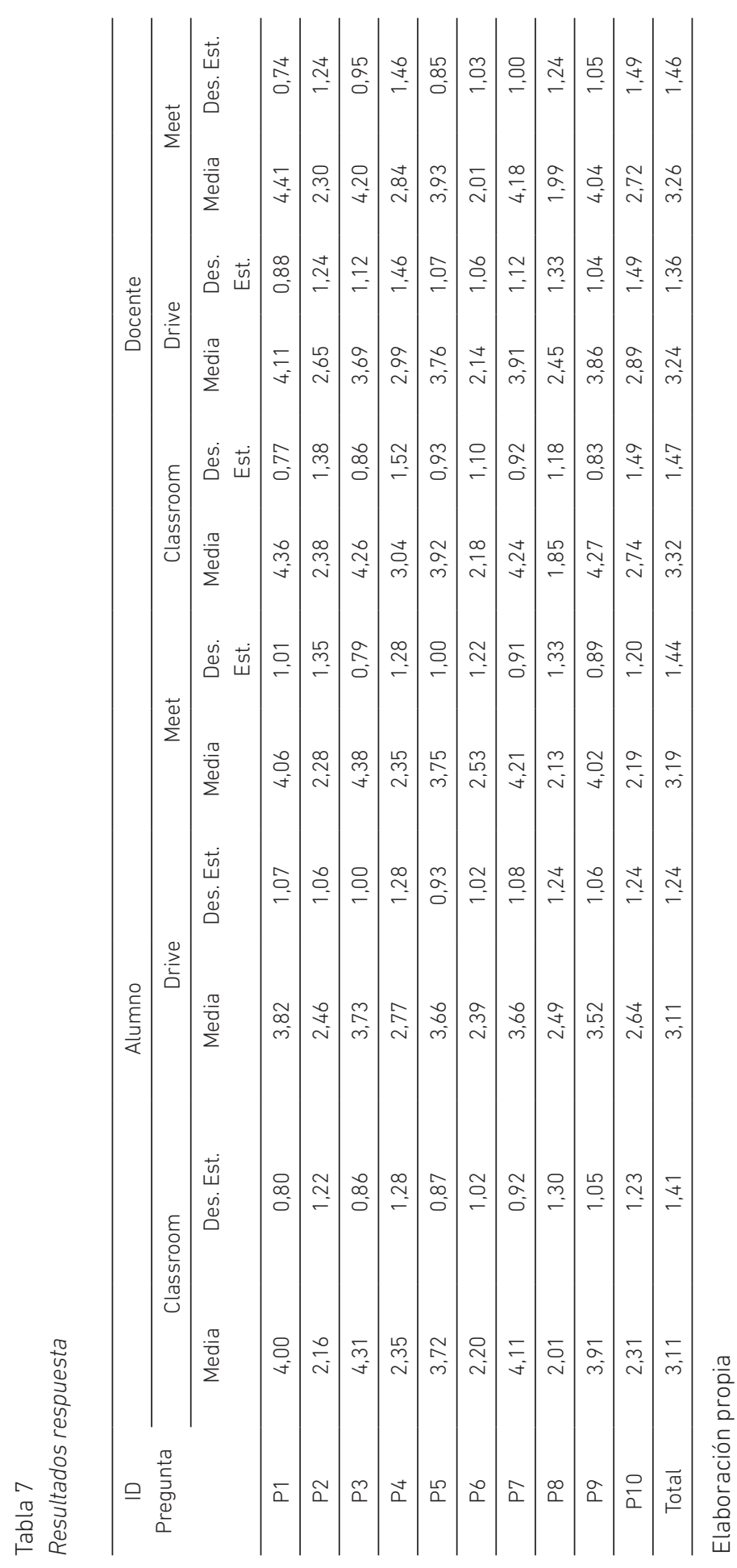


Tabla 8

Resultados puntuación SUS

\begin{tabular}{lcccccc}
\hline & \multicolumn{3}{c}{ Alumno } & \multicolumn{3}{c}{ Docente } \\
\hline \multirow{2}{*}{ Classroom } & Suma & Promedio & Adjetivo & Suma & Promedio & Adjetivo \\
Drive & 9937,50 & 72,50 & Muy bueno & 5340,00 & 72,20 & Muy bueno \\
Meet & 8780,00 & 64,10 & Bueno & 4850,00 & 65,50 & Bueno \\
Total general & 9912,50 & 72,40 & Muy bueno & 5347,50 & 72,30 & Muy bueno \\
\hline
\end{tabular}

Elaboración propia

Tabla 9

Resultados de SUS por escalas

\begin{tabular}{|c|c|c|c|c|c|c|c|c|}
\hline \multirow[b]{3}{*}{ Classroom } & \multicolumn{4}{|c|}{ Alumno } & \multicolumn{4}{|c|}{ Docente } \\
\hline & Usabilidad & Adjetivo & $\begin{array}{c}\text { Capacidad de } \\
\text { aprendizaje }\end{array}$ & Adjetivo & Usabilidad & Adjetivo & $\begin{array}{l}\text { Capacidad de } \\
\text { aprendizaje }\end{array}$ & Adjetivo \\
\hline & 73,97 & $\begin{array}{l}\text { Muy } \\
\text { Bueno }\end{array}$ & 66,79 & Bueno & 77,03 & Muy bueno & 52,70 & Bueno \\
\hline Drive & 65,76 & Bueno & 57,39 & Bueno & 69,05 & Bueno & 51,52 & Bueno \\
\hline Meet & 73,38 & $\begin{array}{l}\text { Muy } \\
\text { bueno }\end{array}$ & 68,25 & Bien & 76,44 & Muy bueno & 55,57 & Bueno \\
\hline $\begin{array}{c}\text { Total } \\
\text { general }\end{array}$ & 71,04 & Bueno & 64,14 & Bueno & 74,17 & Muy bueno & 53,27 & Bueno \\
\hline
\end{tabular}

Elaboración propia

\section{DISCUSIONES Y CONCLUSIONES}

En las tablas 5 y 6 se evidencia claramente que el $68,61 \%$ de los alumnos encuestados utilizan el teléfono celular como uno de los principales dispositivos para acceder a las aplicaciones analizadas en este estudio. En cambio, por parte de los docentes, el 94,5\% de los encuestados hace uso de la laptop como principal dispositivo para el desarrollo de las sesiones de clase. Por lo tanto, existe una clara diferencia en el tipo de dispositivo más utilizado tanto por docentes y alumnos. Esto se debe al rol que desempeñan en el proceso de enseñanza y aprendizaje. Para un alumno resulta más práctico y cómodo acceder a sus sesiones de clase desde un dispositivo móvil porque utiliza funcionalidades de las aplicaciones para captar conocimientos, además, se debe mencionar que el uso del móvil tiene más ventajas de acceso a internet en lugares donde no existe el 
servicio de internet por operadoras. Mientras que, para un docente, que cumple un papel más de guía y mentor, es necesario un dispositivo que le permita aprovechar las funcionalidades orientadas a la compartición de conocimientos.

Por otro lado, el score de SUS obtenido para Google Classroom, Drive y Meet presentados en la tabla 8 , no precisan una diferencia significativa en la usabilidad percibida tanto para profesores y estudiantes. Sin embargo, en el análisis de las subescalas (usabilidad y capacidad de aprendizaje) se observan algunas variaciones, principalmente para la de capacidad de aprendizaje, donde se tiene un menor puntaje para los profesores $(53,27)$ en relación con el de los alumnos $(64,14)$. Esto se debe a que los profesores utilizan más funcionalidades del software que los estudiantes, por ejemplo, deben aprender a publicar anuncios, crear evaluaciones, grabar las clases, enviar y recoger tareas. Esto comprueba, además, que están menos familiarizados con el uso de las tecnologías de la información en comparación con los alumnos por lo que necesitan más tiempo de capacitaciones.

En el caso de los estudiantes que participaron en este estudio el puntaje de SUS para Google Classroom fue de 72,50, sin embargo, en un estudio previo de Alqahtani (2019) el puntaje de SUS para Google Classroom en estudiantes fue de 86,45 , lo que establece una diferencia de 13,95 puntos. Esta diferencia puede ser por el grado de uso de las tecnologías de la información y comunicaciones (TIC) en los distintos países, por ejemplo porque Arabia Saudi tiene una mejor una posición en el Informe sobre la medición de la sociedad de la información 2017 (puesto 54) en comparación con el Perú (puesto 96).

Por otro lado, según Al-Omar (2018), los estudiantes piensan que Blackboard LMS no es fácil de usar y adolece de cierta inconsistencia y complejidad en sus funcionalidades. Este hecho muestra que las herramientas de Google son fáciles de usar, fácil de aprender y menos complejas para adaptar el proceso de enseñanza del ámbito presencial al virtual en el contexto de pandemia del COVID-19.

\section{REFERENCIAS}

Adkins, J. K. (2012). Active Learning with Google Presentations. Journal of Computing Sciences in Colleges, 27(5), 229-230. https://dl.acm.org/doi/ abs/10.5555/2168874.2168924

Ahmad, M. S., Iqbal, M. W., Abid, M., Tabassum, N., Shahzad, S. K., Mian, N. A., y Naqvi, M. R. (2020). Usability Evaluation of Online Educational Applications in COVID-19. LGURJCSIT, 4(4), 86-95. http://lgurjcsit.lgu.edu.pk/index.php/lgurjcsit/article/ view/117/110

Akcil, U., Uzunboylu, H., y Kinik, E. (2021). Integration of Technology to Learning-Teaching Processes and Google Workspace Tools: A Literature Review. Sustainability, 13(9). https://doi.org/10.3390/su13095018 
Al-Omar, K. (2018). Evaluating the Usability and Learnability of the "Blackboard" LMS Using SUS and Data Mining. 386-390. https://doi.org/10.1109/iccmc.2018.8488038

Alqahtani, A. (2019). Usability Testing of Google Cloud Applications: Students' Perspective. Journal of Technology and Science Education, 9(3), 326-339. https://files.eric. ed.gov/fulltext/EJ1215341.pdf

Bardales Mendoza, O., Fernandez Bringas, T., y Saavedra Bendezú, L. (2020). Perspectives of the Use of ICT for the Teaching-Learning Process among Peruvian University Students and Lecturers during the COVID-19Pandemic [Presentación de paper].En 2020 2nd International Workshop on Artificial Intelligence and Education (pp. 49-53). Association for Computing Machinery. https://doi.org/10.1145/3447490.3447500

Brooke, J. (1995). SUS: A “Quick and Dirty” Usability Scale. Usability Evaluation In Industry. https://doi.org/10.1201/9781498710411-35

Cáceres, S. V., y Pow-Sang, J. A. (2018). A systematic mapping review of usability evaluation methods for educational applications on mobile devices [Presentación de paper]. 2018 7th International Conference On Software Process Improvement (CIMPS), 59-68. https://doi.org/10.1109/CIMPS.2018.8625629

Dash, S., Samadder, S., Srivastava, A., Meena R., y Ranjan, P. (2021). Review of Online Teaching Platforms in the Current Period of COVID-19 Pandemic. Indian J Surg. 18, 1-6. doi: 10.1007/s12262-021-02962-4.

de Campos Filho, A. S., de Souza Fantini, W., Ciriaco, M. A., dos Santos, J., Moreira, F., y Gomes, A. S. (2019). Health Student Using Google Classroom: Satisfaction Analysis. En L. Uden, D. Liberona, G. Sanchez, y S. Rodríguez-González (Eds.), Learning Technology for Education Challenges (pp. 58-66). Springer International Publishing. https://doi.org/10.1007/978-3-030-20798-4_6

Fessenden, T. (2016). Net Promoter Score: What a Customer-Relations Metric Can Tell You About Your User Experience. Nielsen Norman Group. https://www.nngroup.com/ articles/nps-ux/

Google for Education. (28 de agosto del 2020).Capacitación sobre aspectos básicos. https:// skillshop.exceedlms.com/student/path/111629-capacitacion-sobre-aspectosbasicos

Hernández Sampieri, R., Fernández Collado, C., y Baptista Lucio, M. del P. (2010). Metodología de la investigación (Quinta). McGRAW-HILL.

Lewis, J. R., y Sauro, J. (2009). The Factor Structure of the System Usability Scale. Lecture Notes in Computer Science (Including Subseries Lecture Notes in Artificial Intelligence and Lecture Notes in Bioinformatics), 5619 LNCS, 94-103. https://doi. org/10.1007/978-3-642-02806-9_12 
Lewis, J. R., y Sauro, J. (2018). Item Benchmarks for the System Usability Scale. Journal of Usability Studies, 13, 158-167. https://uxpajournal.org/item-benchmarkssystem-usability-scale-sus/

Lewis, S. G., y Kimmel-Smith, S. (2011). Orchestrating an Institution-Wide Transition to Google Calendar. Proceedings of the 39th Annual ACM SIGUCCS Conference on User Services, 95-102. https://doi.org/10.1145/2070364.2070388

Lindoo, E. (2009). Using Google Sites, Google Groups and Google Documents to Enhance Your Course. Journal of Computing Sciences in Colleges, 25(2), 46-51. https:// dl.acm.org/doi/abs/10.5555/1629036.1629043

Mavri, A., y Hadjiconstantinou, S. (2018). Evaluating the Use of Groupware Technologies in Support of Collaborative Learning in an ESP Tertiary Education Course. En P. Zaphiris y A. Ioannou (Eds.), Learning and Collaboration Technologies. Design, Development and Technological Innovation (pp. 446-465). Springer International Publishing.

Mendoza, S. H. V. (2015). Improving Communication and Building Communities with Google [Presentación de paper]. Proceedings of the 2015 ACM SIGUCCS Annual Conference, 85-90. https://doi.org/10.1145/2815546.2815574

Nielsen, J. (1994). Usability Engineering. Elsevier Science. https://books.google.com.pe/ books?id=95As20F67f0C

Nina, H., Enciso, L., y Chavez, W. A. (2017). Software as a Service Google Apps in the Internal Communication of the National University of San Antonio Abad del Cusco [Presentación de paper]. Proceedings of the International Conference on Industrial Engineering and Operations Management Bogota, Colombia, October 25-26, 2017, 701-710. http://ieomsociety.org/bogota2017/papers/113.pdf

Nina, H. (2019). Implementación de un nuevo modelo de servicio computacional para mejorar la comunicación interna Universitaria Nacional de San Antonio Abad del Cusco [Tesis de Doctorado, Universidad Nacional Federico Villarreal]. Repositorio Institucional de la Universidad Nacional Federico Villarreal. http://repositorio. unfv.edu.pe/handle/UNFV/3335

Paz, F., y Pow-Sang, J. A. (2016). A systematic mapping review of usability evaluation methods for software development process. International Journal of Software Engineering and Its Applications, 10(1), 165-178. https://www.researchgate. net/publication/297764945_A_systematic_mapping_review_of_usability_ evaluation_methods_for_software_development_process

Plataforma digital única del Estado Peruano. (11 de agosto del 2021). Normatividad sobre coronavirus (COVID-19). https://www.gob.pe/institucion/minsa/colecciones/749normatividad-sobre-coronavirus-covid-19 
Rusmansyah, Hayati, N., Winarti, A., y Rahmi. (2021). Train Students' Science Process Skills and Self-Efficacy in Online Learning Using the Scientific Critical Thinking (SCT) Model Assisted by Google Classroom and Google Meet. Journal of Physics: Conference Series, 1760, 012034. https://doi.org/10.1088/1742$6596 / 1760 / 1 / 012034$

Sauro, J. (19 de septiembre del 2018). 5 Ways to Interpret a SUS Score - MeasuringU. https://measuringu.com/interpret-sus-score/

Sauro, James Lewis, J. R., New York, L., Diego, S., y Kaufmann, M. (2012). Quantifying the User Experience Practical Statistics for User Research (2nd Ed.).

Septantiningtyas, N., Juhji, J., Sutarman, A., Rahman, A., Sa'adah, N., y Nawisa. (2021). Implementation of Google Meet Application in the Learning of Basic Science in the COVID-19 Pandemic Period of Student Learning Interests. Journal of Physics: Conference Series, 1779, 012068. https://doi. org/10.1088/1742-6596/1779/1/012068

SUNEDU. (2019). Informe Técnico de Licenciamiento N. ${ }^{\circ}$ 011-2019-SUNEDU/02-12. https:// intranet.sunedu.gob.pe/documentos/directorios/320/res-056-2019-suneducd-resuelve-otorgar-licencia-institucional-a-unsaac--28itl-29-comprimido.pdf

UNESCO. (2021). COVID-19: Reopening and reimagining universities, survey on higher education through the UNESCO National Commissions. https://unesdoc.unesco. org/ark:/48223/pf0000378174.locale=en

Unión Internacional de Telecomunicaciones. (2017). Informe sobre la Medición de la Sociedad de la Información del 2017. https://www.itu.int/en/ITU-D/Statistics/ Pages/publications/mis2017.aspx

Usability.gov. (25 de Julio del 2021). System Usability Scale (SUS). https://www.usability. gov/how-to-and-tools/methods/system-usability-scale.html

Widiyatmoko, A. (2021). The Effectiveness of Google Classroom as a Tool to Support Online Science Learning: A Literature Review. Journal of Physics: Conference Series, 1918(5), 052069. https://doi.org/10.1088/1742-6596/1918/5/052069 